\title{
Matrix-Based Key Management Scheme for IoT networks
}

\author{
Mohammed Nafi ${ }^{\mathrm{a}}$, Samia Bouzefrane ${ }^{\mathrm{b}}$, Mawloud Omar ${ }^{\mathrm{c}}$ \\ ${ }^{a}$ Laboratoire d'Informatique Médicale, Faculté des Sciences Exactes, Université de Bejaia,06000 Bejaia, Algérie \\ ${ }^{b}$ CEDRIC Lab, Conservatoire National des Arts et Métiers-CNAM, Paris, France \\ ${ }^{c}$ LAMOS, Faculté des Sciences Exactes, Université de Bejaia,06000 Bejaia, Algérie
}

\begin{abstract}
The key management is the central element of network security. In fact, key distribution is necessary for securing applications in the context of Internet of Things (IoT). However, existing key management protocols are not directly applicable on IoT due, among other things, to severe and high resource constraints of some devices that make up the IoT network. Therefore, it is necessary that the proposed key management protocols takes in charge these features and constraints. Most existing solutions didn't focus on optimizing, at the same time, all performance criteria, like communication, computation and storage. Some of them put special emphasis on minimizing one criteria but ignore the others. In this paper, we propose a new lightweight matrix based key management protocol for Iot network, which is not only flexible, scalable and resilient to many types of attacks, but also can reduce the communication, computation and storage overheads at constrained nodes side. The security properties like authentication, integrity and secrecy have been checked by using the formal verification tool AVISPA. Moreover, security and performance analysis show that our scheme protects user's sensitive data from several types of attacks by achieving secure end-to-end communications, and optimizes the energy consumption, which is suitable for resource-limited networks.
\end{abstract}

Keywords: Key management, Security, Internet of Things, Dynamic networks

\section{Introduction}

The concept of Internet of Things (IoT) was initially introduced by Kevin Ashton in 1999 [1]. The IoT system is made up of various types of heterogeneous devices, called objects or things. These objects are interconnected and have the capability to communicate with each other and with their environment. IoT devices can be accessible and managed at anytime and anywhere, since they are connected to the Internet through an Internet Protocol (IP) router [2]. Sensors, actuators and RFID tags for instance, have the ability to sense their environment by acquiring measurements, to store and process the gathered data, and communicate with each other by exchanging data over the network. This interaction between devices is performed without human intervention [3].

To make the communication between these IoT devices more secure, a key management service is needed. However, key management in the context of IoT is more challenging than in traditional networks. This is because of many reasons such as the use of wireless links that are vulnerable to eavesdropping attacks; lack of a central authority; mobility and heterogeneity of devices in terms of resources such as memory, computing and energy capacity; high resource constraints of many devices, like sensors that have limited battery, computation and memory capability; accessibility to the objects via the Internet, which exposes them to several types of attacks, in particular denial-of-service (DoS), etc. For all these reasons, integrating security for IoT system is a real challenge and new key management solutions must take in charge these inherent features.

In order to meet these challenges, several key management approaches have been proposed in the literature for securing IoT networks, which can be classified into two main categories: preshared and public key approaches. The main drawback of most of them is they focus primarily on optimizing some 
performance criteria, but completely neglect the others. For instance, some protocols put special emphasis on decreasing communication overhead, but ignore the storage and/or the computation costs.

In this paper, we propose a new lightweight key management scheme for Iot networks that is based on matrix, which is not only flexible, scalable and resilient to many types of attacks, but also can considerably reduce the amount of information to be exchanged and processed at constrained nodes side without ignoring the storage overhead.

The main contributions of this paper are the following:

- The number of keys to be stored in node's memory is very small since a node stores at most only the pairwise keys of its direct neighbors with the group key;

- The approach offers a negligeable communication overhead. In other words, no much communication is required during key establishment phase because the keys are generated in a distributed manner.

- Low computation cost because no complex operation is required to establish secret keys so the computation of symmetric keys betwen two neighbors is efficient.

- The proposed approach is flexible since it allows the addition of new devices to the network after the deployment phase.

- The security goals such as secrecy, integrity and authentication are guaranteed.

- The proposed scheme is resistant to several types of attacks such as eavesdropping, compromising, sybil, forward, backward and replay attacks.

- The secret keys are established in a distributed manner. Therefore, it avoids a central entity that could be a point of failure or weakness.

- The proposed scheme allows gateway and constrained nodes belonging to the same group to communicate securely by using one group key.

- The security analysis and performance evaluation show that the proposed scheme can protect user's data privacy and saves energy which is suitable for the resource-limited network.

The organization of the rest of this paper is as follows. Related work on IoT security and key management is summarized in Section 2. In Section 3, we present the proposed matrix based key management scheme, which consists of a set of protocols for initialization, pairwise and group key establishment, node addition, key revocation and periodic key renewal phase. In Section 4, we describe both informal and formal analysis of our protocol. Section 5 presents the performance analysis wherin a comparative and an experimental studies are described. Finally, Section 6 concludes the paper and gives future directions.

\section{Related work}

According to [4], the key management protocols proposed in the literature for IoT networks can be classified into two main categories: (1) preshared approaches, which are based on the predistribution of shared context that is used to generate a secret shared key between two communicating entities; and (2) public key approaches, which are based on asymmetric encryption to generate a commun secret between two entities having no previous preshared context.

Among the schemes belonging to the first category, we find a matrix-based scheme for establishing pairwise keys that was initially proposed by Blom [5]. This scheme allows any pair of nodes to be able to compute a commun pairwise key. It is based on the use of a set of matrices : a $(\lambda+1) \cdot N$ public matrix, called $\mathrm{G}$, known to everyone, a $(\lambda+1) \cdot(\lambda+1)$ random symmetric matrix, called $\mathrm{D}$, and a $N \cdot(\lambda+1)$ secret matrix, called $A=(D \cdot G)^{T}$. Since A is a symmetric matrix, the key matrix $K=A \cdot G$ is also symmetric. Therefore, the element $(i, j)$ of $\mathrm{K}$ is equal to the element $(j, i)$ that correspond to the pairwise key shared 
betwen the nodes $i$ and $j$. Every node $i$ maintains in its memory the $i$ th row and $i$ th column of the private and public matrix respectively as key material. When two nodes $i$ and $j$ want to communicate, they first exchange their public column vector of $\mathrm{G}$ then each node calculates separately the same commun key $K_{i j}=K_{j i}$, which is obtained by the product of the private row vector of A of one node and the public column of the other node. In this scheme, since nodes never exchange their private rows, no adversary can compute any pairwise key by simply listening to the communications. In addition, it has been shown in [5] that the above scheme is $\lambda$-secure when all the columns of the matrix $G$ are linearly independent. However, if the number of compromised nodes exceeds the threshold $\lambda$, the whole secret matrix $\mathrm{K}$ could be calculated, thus the entire network becomes unsecure.

Based on the above Blom's scheme, Du et al. [6] proposed a new key pre-distribution scheme that uses multiple-space key in order to acheive better resilience of the network to node capture attack. In fact, to be able to break at least one key space, an adversary must capture a significant number of nodes equals to $\lambda+1$ that all share the same key space's information. In this scheme, every node needs to store a row vector of $\lambda+1$ elements of its private information for each selected key space and a single element (a seed) of its public information. To be able to compute a commun pairwise key, two nodes must share at least one key space. Compared to Blom's scheme, this scheme is more resilient to node capture attack and more efficient in terms of communication, but less in terms of computation and storage. In fact, nodes do not need to exchange their public column, which reduces the communication overhead, but it is regenerated by the node itself, which increases computation overhead (2 modular multiplications). Moreover in blom's scheme, a node needs only to store $2(\lambda+1)$ elements, but here it stores a seed and $\lambda+1$ elements for each key space, so $(\lambda+1) \cdot t$, where $t$ is the number of key spaces. Furthermore, this scheme does not address node addition, revocation and key refresh.

$\mathrm{Yu}$ and Guan in [7]. proposed a key management scheme using deployment knowledge based on Blom's scheme. In their protocol, a deployment area is partionned into grids (hexagons, squares or triangles) and sensor nodes are arranged into groups where the number is the same as that of grids. After that, each group of sensors is deployed into a single grid. The authors show that hexagonal grids are best in terms of security and memory requirement compared with the others. During key predistribution phase, all groups share the same global matrix $\mathrm{G}$ and each one is assigned a unique secret matrix $\mathrm{A}$ and a set of $B$ matrices. During discovery phase, each pair of neighbors that belong to the same group, generate the pairwise key from the common matrix A and G as in Blom's scheme. The nodes that are not from the same group but share at least one common B matrix, can also compute a common key from one choosen matrix B and the matrix G using Blom's scheme too. The other nodes that can not compute pairwise keys between them may use another key discovery mechanism to establish pairwise keys. In this scheme, each node stores one column of matrix $\mathrm{G}$, one row of matrix $\mathrm{A}$, and, at most, $w$ rows of $\mathrm{B}$ matrices, where each row has $\lambda+1$ elements. Moreover, if more than $\lambda$ nodes of a group are compromised, the matrix A and some B matrices will be broken. However, their scheme achieves higher connectivity with much lower memory requirement and shorter transmission range. Furthermore, it is more resilient against node capture attack.

Another scheme based on Blom's scheme has been proposed by Rahman et al. [8]. The authors have improved the original Blom's scheme so that it becomes suitable for use in resource-constrained environment like wireless sensor networks. In this scheme, two nodes are able to generate a common key without any messages exchange between them, but by knowing only the identifier of the other. In fact, a node does not need to store or exchange its public column of the matrix G, but generates it by using the node's identifier, which significantly reduces the storage and the communication overhead. In addition, the authors proposed mechanisms for updating keys, adding new nodes after deployment, and revoking compromised nodes. They also presented a dynamic mechanism for establishing secure group keys that uses pairwise keys. This makes their model more flexible. However, the public matrix $\mathrm{G}$ is generated by the node itself, which increases the computation overhead on the node side, and affects the resilience of the scheme to node capture attack. Indeed, when an attacker compromises a single node, he is not only able to compute all its keys shared with the other nodes, but also obtains more information about the 
secret matrix.

The authors Y. Zhang et al in [9]. have presented a matrix-based cross-layer key establishment protocol for smart homes with no prior secret sharing. This is motivated by the fact that domestic devices are heterogeneous and are not necessarily produced by the same factory. The protocol is based on [10] and uses the multiple key-spaces idea proposed in [6]. Two kinds of keys are used in this protocol: the secret master key $k i$ and the secret session key $K i j$. The first key is extracted by using the physical layer key extraction algorithm [11] when the device joins the network, and shared between a device and a home gateway. The second one is established at higher levels and shared between two appliances $P i$ and $P j$. Thus, the proposed protocol allows any pair of devices to be able to compute a common secret session key with light energy consumption by delegating the heavy operations to the home gateway that is powerful. It also achieves key refresh and network scalability. In addition to this, the storage cost is very low since home appliances have no need to pre-load any secrets and store only the key seed sent by the home gateway. However, each appliance needs to compute $2 \lambda+1$ multiplications to establish a session key and the communication cost of the proposed protocol is relatively higher compared with the previous ones.

Messai and Seba in [12]. presented a new key management scheme for hierarchical wireless sensor networks, called EAHKM+. This scheme is composed of two phases: the key pre-distribution phase and the cluster formation and key establishment phase. In the first phase, each sensor node is pre-assigned with three keys before its deployment in the sensing environment: the network key known to all sensor nodes in the network, which is deleted after the second phase, and two pairwise keys used to secure the communication channel that separates sensor node from the base station. In the second phase, clusters are securely formed and two new keys are established: the cluster key shared between all cluster members and a pairwise key shared between the sensor node and its cluster head. This scheme provides a secure cluster creation and it is flexible since it allows adding and removing sensor nodes after deployment. In addition to this, it is scalable and can support a large number of nodes. However, the rekeying process is costly in terms of communication and computation since it requires the re-run of clustering algorithm.

S. Mesmoudi et al in [13] have quite recently, proposed a smart and dynamic key management scheme for hierarchical wireless sensor networks, called SKWN. The scheme includes key establishment, key renewal and new node addition. The proposed protocol is based on machine learning to supervise the network activity and choose the appropriate security level. It particularly uses ISA component to detect intrusions that occur in the network and deal with in real time. It is therefore not necessary to use a high security level in the absence of intruder. SKWN is scalable, flexible and provides reliable security mechanism. However, the number and the size of exchanged messages during different phases remain high.

To overcome the limitations of current key distribution and management approaches, we propose an efficient and a new matrix-based key management scheme.

\section{Overview of the proposed scheme}

In this section, we describe our matrix-based key management system for Iot networks that aims to minimize the computation, communications and storage overhead. First of all, we begin by presenting the network model that we are considering, then we give some assumptions. After that, we summarize the notations used in this paper. Finally, we describe in detail the differents phases of our scheme.

\subsection{Network model}

We adopt the network architecture that consists of three main components: constrained nodes, gateway nodes and remote server node (command node), as shown in Figure 1.

\subsubsection{Constrained nodes}

In this category, we can find all nodes that are highly constrained in terms of resources (energy, memory and computation), such as sensors, RFID Tags, wearable devices (like watch) that can be carried 
by a human, etc. The role of these devices consists of monitoring or sensing the environment so that they collect and transmit the collected data to gateway nodes via bluetooth, ZigBee or wifi technologies. For example, in healthcare application, sensors can be planted in or on a human's body in order to collect health related data (e.g. blood pressure, blood glucose level, temperature level, etc.). Another examples of constrained nodes are MICAz and TelosB that are really constraining. Both platforms run TinyOS. The MICAz is based on the low-power 8-bit microcontroller ATmega128L running at $7.37 \mathrm{MHz}$ and the TelosB is based on the 16-bit microcontroller running at $4 \mathrm{MHz}$ [14].

\subsubsection{Gateway nodes}

The gateway nodes have significantly higher energy resources and are equipped with high performance processors and more memory compared to nodes belonging to the previous category (constrained nodes), but they have less resources when compared to remote server node. The gateway nodes fuse the received data collected by the different constrained nodes, process and send or forward it to the remote server (command node). The communication between the gateway node and the constrained nodes may be via Bluetooth or ZigBee, and the one between the gateway node and the remote node may be done through cellular $(3 \mathrm{G}, 4 \mathrm{G})$ or WiFi networks.

\subsubsection{Remote server node (command node)}

It can be assumed that the server node has no limitations in terms of power, computation and storage compared to the two previous ones. For instance, the medical specialists or doctors at remote server node side can continuously follow patient's health status based on the data received in order to intervene in time.

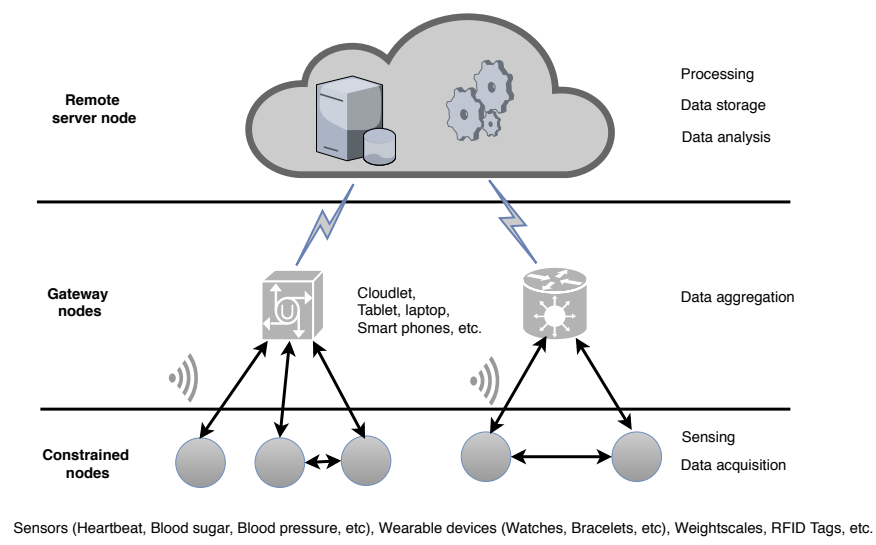

Figure 1: Network Model

\subsection{Assumptions}

- We have not made any trust assumptions about the constrained nodes. They can therefore be captured and compromised. This means that the adversary can read all the information from the node's memory, including the keys.

- Constrained nodes are only able to perform symmetric encryption to avoid high computation since they are limited in resources.

- We assume that the gateway nodes can also be captured by the attacker and are supposed to be able to perform symmetric and asymmetric encryption. So, we propose to include TPM (Trusted Platform Module) $[15,16]$ to secure the keys stored in their memory.

- The remote server node is powerful enough to support symmetric and asymmetric cryptography.

The notations used in this paper are listed in Table 1. 


\begin{tabular}{ll}
\hline Notation & Description \\
\hline$I d i$ & Identification number of the node $N i$. \\
$M$ & Square matrix of order $n$, where $n$ refers to the size of the network \\
& during the deployment \\
$M_{i}$ & Matrix of the node $N i$ \\
$v i$ & Neighbors vector of the node $N i$ \\
$a \| b$ & Information $a$ is concatenated with $b$ 's one \\
$H a s h(M s g, k)$ & One way keyed-hash function \\
$|x|$ & Absolute value of the number $x$ \\
$\operatorname{det}(M)$ & Determinant of the matrix $M$ \\
$S_{i j}$ & Positive secret value computed by two neighbors $N i$ and $N j$ \\
$K_{i j}$ & Pairwise secret key shared betwen two neighbors $N i$ and $N j$ \\
$K_{i} / K_{i}^{-1}$ & Public/Private key of the gateway node $G N i$ \\
$K_{g i}$ & Group key generated by the gateway node $G N i$ \\
$S i$ & Random positive secret value generated by the gateway $G N i$ \\
$\{M\} k$ & Message $M$ is encrypted with the key $k$ \\
$T i$ & Timer of the node $N i$ \\
$N o n c e_{i}$ & Nonce (random value) generated by the node $N i$ \\
$L i s t_{i}$ & List kept by the node $N i$ \\
\hline
\end{tabular}

Table 1: Notations

\subsection{Phases of our scheme}

The proposed scheme is composed of six phases: initialization, pairwise and group key establishment, new node addition, key revocation and key refresh phase. These phases are described in detail in the following section.

\subsubsection{Initialization phase}

During this phase, all of the following information is preloaded into nodes' memories just before their deployment in the network:

1. An identification number $I d i$ that is unique in the network.

2. A square matrix $M$ of order $n$, where the elements are generated randomly and strictly positives, and $n$ represents the number of nodes in the network during the deployment phase: $M[i, j]>0$, with $i, j=1 . . n$. This matrix can be stored in the flash RAM and hence will be erased from the nodes' memories later.

3. A one way keyed-hash function $\operatorname{Hash}(m s g, k)$ that takes as input a message of arbitrary length $m s g$ and a secret key $k$. The initial message will be compressed into a short fixed length hash value, so it will take up less space.

With the matrix $M$ and the hash function $\operatorname{Hash}()$, every node $N i$ is able to compute the symmetric key $K_{i j}$ that will be shared with its direct neighbor $N j$. Moreover, each gateway node has a timer initialized to a value which decrements with time. When it reaches zero, the key renewal process will be launched.

\subsubsection{Pairwise key establishment phase}

The pairwise key generation phase contains the two following steps: 
a) Neighbor discovery

As soon as the deployment is done, every node $N i$ identifies its direct neighbors (1-hop) by sending them a Helloi message along with its identifier, a nonce and a hash value (digest), which is computed by the preshared one way function that uses the diagonal element of the sender as secret value.

$$
N i \rightarrow G N j: \text { Helloi, Idi } \| \text { Nonce }_{i}, \operatorname{Hash}\left(\operatorname{Idi} \| \text { Nonce }_{i}, M[i, i]\right)
$$

Upon the reception of this message, the recipients that are within the radio range of the sender check the authenticity and integrity of the message. In fact, they calculate the hash value $H$ of the first part of the received message by using the diagonal element $M[i, i]$ of the sender as secret value as follows: $H=\operatorname{Hash}\left(\operatorname{Idi} \|\right.$ Nonce $\left._{i}, M[i, i]\right)$. The obtained result will then be compared to the second part ie $\operatorname{Hash}\left(I d i \|\right.$ Nonce $\left._{i}, M[i, i]\right)$, which corresponds to the hash value computed by the sender node $N i$. If both digests are different, then this message will be ignored and rejected, otherwise it is authentic, so it has not been altered by an adversary during its transmission. As a result, this message will be accepted by the recipient node and the latter updates its neighbors vector $v j$ by adding the identifier $I d i$ of $N i$ and arranges the vector in ascending order. After that, the gateway node replies to the sender node $N i$ with the Helloj message that contains its identifier, the succesor of the received nonce and a digest.

$$
G N j \rightarrow N i: H_{e l l o j} \text { Idj } \| \text { Nonce }_{i}+1, \operatorname{Hash}\left(\operatorname{Idj}_{\|} \| \text {Nonce }_{i}+1, M[j, j]\right)
$$

At the end of this step, each node has its neighbors vector completely updated that contains all the identifiers of its 1-hop neighbors arranged in ascending order.

b) Pairwise key computation

During this step, each node computes its square matrix $M_{i}$, which is obtained from the initial one $M$ by keeping only the elements $M[k, l]$, where $\forall \quad k, l \in v_{i}$.

Moreover, it computes a positive secret value $S_{i j}$, which is equal to the absolute value of the determinant of the 2 by 2 matrix $M i j$, where the elements are:

$$
\begin{aligned}
M i j=\left[\begin{array}{ll}
M_{i}[i, i] & M_{i}[i, j] \\
M_{i}[j, i] & M_{i}[j, j]
\end{array}\right] & =\left[\begin{array}{ll}
M_{j}[i, i] & M_{j}[i, j] \\
M_{j}[j, i] & M_{j}[j, j]
\end{array}\right], \text { with } i<j \\
S_{i j} & =|\operatorname{det}(M i j)|
\end{aligned}
$$

Finally, it obtains a shared secret key $K_{i j}$ by applying the hash function that uses $S_{i j}$ as secret value as follows:

$$
K_{i j}=\operatorname{Hash}\left(I d i\|I d j\| S_{i j}, S_{i j}\right), \quad \text { with } \quad i<j
$$

This pairwise key will be used by the two nodes in order to secure their communications by encrypting and decrypting the messages exchanged between them. Note that keys can or not be stored in nodes' memories according to their capacity. In fact, a node with low storage and high processing capacity may store few or no keys. The other keys will be calculated during communication, which reduces storage costs. On the other hand, a node with high storage and low processing capability can store all or a large number of pairwise keys. This allows the node to avoid recalculating them during the next communication session with the same node, which reduces the calculation cost. Thus, a node can store a set of keys whose number is between one and $d+1$ where $d$ is the number of its neighbors.

In addition, each gateway node $G N i$ computes its private/public keys $K_{i}^{-1} / K_{i}$ that will be used to secure the communication channel separated him to the server node. The private key is computed as follows:

$$
K_{i}^{-1}=\operatorname{Hash}\left(S_{i j}\left\|S_{i k}\right\| \ldots \| S_{i l}, M[i, i]\right)
$$

where $j, k, . ., l \in v_{i}$ and $j<k<. .<l$.

The public key is obtained from the last private key as follows: 


$$
K_{i}=\operatorname{Hash}\left(K_{i}^{-1}, M[i, i]\right)
$$

The key $K_{i}$ is public and known by everyone. It can be specially used by the server node when he wants to send messages securely to that gateway node. However, the key $K_{i}^{-1}$ is private and must be kept secret and known only by the gateway node itself. It can be used to sign/decrypt messages sent/received to/from the server node.

After the computation of the public keys, each gateway node computes the hash value $H=\operatorname{Hash}(\operatorname{Id} i \|$ $\left.K_{i} \| M[i, i], M[i, i]\right)$ and puts the couple $\left(K_{i}, H\right)$ in its public repository so that the server node can have access to it. When the latter intends to communicate with that gateway node, it takes the first part of the couple, namely the public key $K_{i}$, and computes the hash value $H^{\prime}$ by using the identity, the first part of the couple (public key), and the corresponding diagonal element of the initial square matrix. The result is then compared with the second part of the couple, namely $H$. If the verification is successful, the server node stores this public key in its memory, otherwise, the public key is not authentic so it will be ignored. The server node sends its public key encrypted with the authentic public key of each gateway node. These latter use their private key so as to decrypt the message and stores the public key of the server.

The complete process of establishing of the symmetric key between the gateway node $j$ and its direct neighbor $i$ is described in the Figure 2 below.

Constrained Node i

Gateway Node j

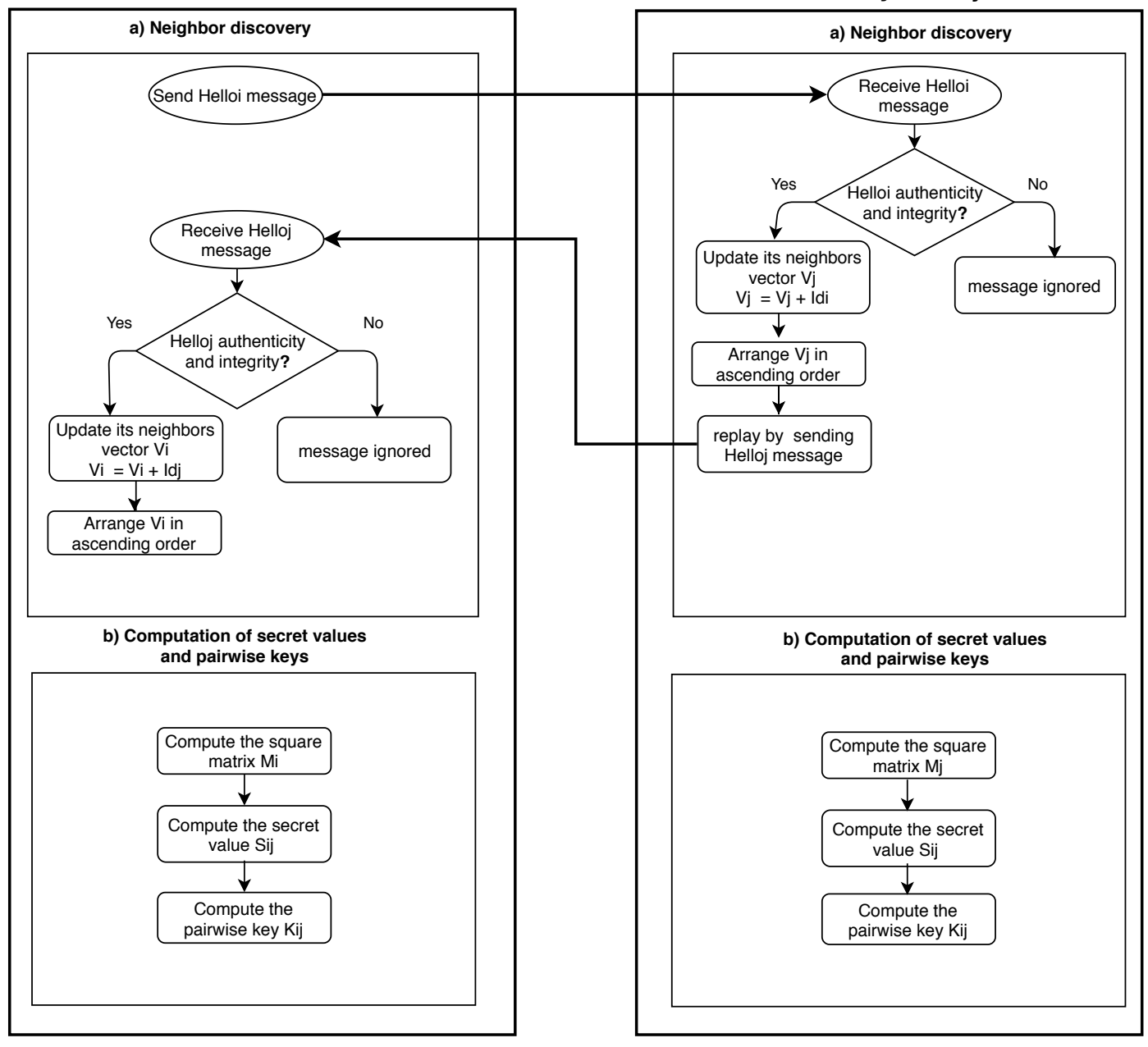

Figure 2: Process of establishing of the pairwise key between two nodes 


\subsubsection{Group key establishment phase}

The group communication can significantly improve performance and energy efficiency. In fact, when nodes intend to send or broadcast the same data to a set of devices in a secure manner, it is suitable to use one key, called group key. Thus, all group members share and use this common secret key in order to secure their communications within the group. In our scheme, these group keys are generated by gateway nodes since they are trustworthy and have more resources. These keys are then distributed to group members in a secure manner. Indeed, each gateway node randomly chooses one pairwise key $K_{i r}$ among these it shares with its direct neighbors, and use it as input to the hash function in order to generate one group key as follows:

$$
K_{g i}=\operatorname{Hash}\left(K_{i j}\left\|K_{i k}\right\| \ldots \| K_{i l}, K_{i r}\right)
$$

where $j, k, . ., l, r \in v_{i}$

Every gateway node sends the generated group key along with a nonce, to each of its direct neighbors encrypted with the appropriate pairwise key.

$$
G N i \rightarrow N j:\left\{K_{\text {gi }}, \text { Nonce }_{i}\right\} K_{i j}
$$

Upon receiving the last message, each group member uses the appropriate pairwise key to decrypt it. Then it stores the received group key in its memory and reponds with the following acknowledgement message.

$$
N j \rightarrow G N i:\left\{\text { Ack } \text { Nonce }_{i}+1\right\} K_{i j}
$$

This way, all nodes that are neighbors of the same gateway node form a group and can use the group key to secure their communications within the group.

\section{Example}

The network depicted in the Figure 3 is composed of four nodes: three sensors and one gateway node. During the initialization phase, all nodes are assigned a unique identifier(number) and pre-loaded with the hash function $\operatorname{Hash}()$ and the square matrix $M$ of order 4 (a 4 by 4 matrix).

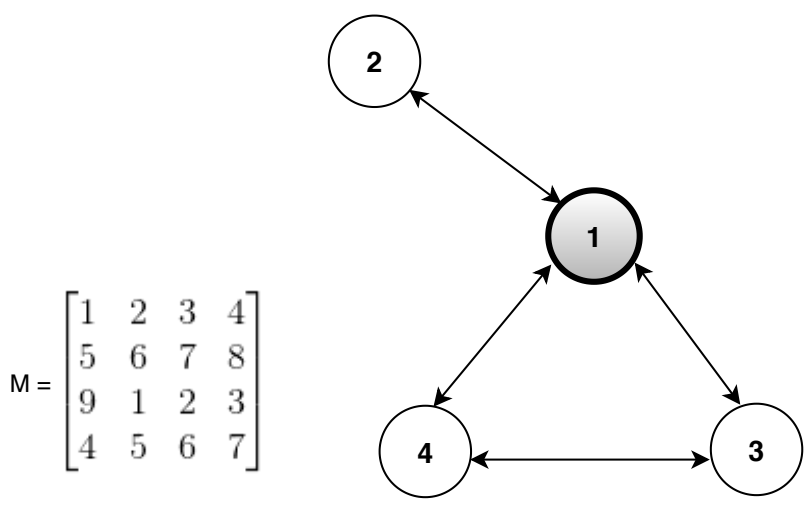

Figure 3: Network with four nodes

During the neighbor discovery stage, the nodes of the network exchange hello messages betwen them so that they update their neighbors vector and compute their square matrix as follows:

The gateway node 1 has three neighbors (nodes 2, 3 and 4). Therefore, its neighbors vector $v 1$ has four identifiers arranged in ascending order (its identifier and the identifier of each neighbor). So the vector $v 1=[1,2,3,4]$ and its square matrix $M 1$ is equal to the initial matrix $M$ because all the nodes are neighbors with the node 1 . 
The node 2 has one neighbor (node 1). Hence, its neighbors vector $v 2$ has two identifiers (the identifier of the node 1 and its identifier in this order) arranged in ascending order $v 2=[1,2]$ and its square matrix $M 2$ is obtained as follows:

$$
M 2=\left[\begin{array}{ll}
M[1,1] & M[1,2] \\
M[2,1] & M[2,2]
\end{array}\right]=\left[\begin{array}{ll}
1 & 2 \\
5 & 6
\end{array}\right]
$$

The nodes 3 and 4 have the same neighbors (nodes 1,3,4), thus they have the same neighbors vector $v 3=v 4=[1,3,4]$ and they share the same 3 by 3 matrix $(M 3=M 4)$ obtained from the matrix $M$ as follows :

$$
M 3=M 4=\left[\begin{array}{lll}
M[1,1] & M[1,3] & M[1,4] \\
M[3,1] & M[3,3] & M[3,4] \\
M[4,1] & M[4,3] & M[4,4]
\end{array}\right]=\left[\begin{array}{lll}
1 & 3 & 4 \\
9 & 2 & 3 \\
4 & 6 & 7
\end{array}\right]
$$

In the next step, each pair of neighbors compute the secret value in the following way:

The nodes 1 and 2 compute the secret value:

$$
S 12=|\operatorname{det}(M 12)|=\left|\operatorname{det}\left(\begin{array}{cc}
M[1,1] & M[1,2] \\
M[2,1] & M[2,2]
\end{array}\right)\right|=\left|\operatorname{det}\left(\begin{array}{cc}
1 & 2 \\
5 & 6
\end{array}\right)\right|=|6-10|=4
$$

The nodes 1 and 3 compute the secret value:

$$
S 13=|\operatorname{det}(M 13)|=\left|\operatorname{det}\left(\begin{array}{cc}
M[1,1] & M[1,3] \\
M[3,1] & M[3,3]
\end{array}\right)\right|=\left|\operatorname{det}\left(\begin{array}{ll}
1 & 3 \\
9 & 2
\end{array}\right)\right|=|2-27|=25
$$

The nodes 1 and 4 compute the secret value:

$$
S 14=|\operatorname{det}(M 14)|=\left|\operatorname{det}\left(\begin{array}{cc}
M[1,1] & M[1,4] \\
M[4,1] & M[4,4]
\end{array}\right)\right|=\left|\operatorname{det}\left(\begin{array}{ll}
1 & 4 \\
4 & 7
\end{array}\right)\right|=|7-16|=9
$$

The nodes 3 and 4 compute the secret value:

$$
S 34=|\operatorname{det}(M 34)|=\left|\operatorname{det}\left(\begin{array}{cc}
M[3,3] & M[3,4] \\
M[4,3] & M[4,4]
\end{array}\right)\right|=\left|\operatorname{det}\left(\begin{array}{ll}
2 & 3 \\
6 & 7
\end{array}\right)\right|=|14-18|=4
$$

Finally, in the last stage, every two neighboring nodes are able to generate the common pairwise key as follows:

The nodes 1 and 2 compute the secret symmetric key:

$K 12=\operatorname{Hash}(1\|2\| 4,4)=\operatorname{Hash}(124,4)$

The nodes 1 and 3 compute the secret symmetric key:

$K 13=\operatorname{Hash}(1\|3\| 25,25)=\operatorname{Hash}(1325,25)$

The nodes 1 and 4 compute the secret symmetric key:

$K 14=\operatorname{Hash}(1\|4\| 9,9)=\operatorname{Hash}(149,9)$

The nodes 3 and 4 compute the secret symmetric key:

$K 34=\operatorname{Hash}(3\|4\| 4,4)=\operatorname{Hash}(344,4)$

The gateway nodes 1 also computes its private/public keys as follows:

$K_{1}^{-1}=\operatorname{Hash}\left(S_{12}\left\|S_{13}\right\| S_{14}, M[1,1]\right)=\operatorname{Hash}(4253,1), K_{1}=\operatorname{Hash}\left(K_{1}^{-1}, M[1,1]\right)$.

Moreover, it randomly chooses a pairwise key among $K_{12}, K_{13}$ and $K_{14}$, let's say $K_{12}$, and computes the group key $K_{g 1}$ as follows:

$K_{g 1}=\operatorname{Hash}\left(K_{12}\left\|K_{13}\right\| K_{14}, K_{12}\right)$.

This group key is then transmitted to each group member within message encrypted with the appropriate pairwise key. In fact, the messages $\left\{K_{g 1}\right.$, Nonce $\left._{1}\right\} K_{12},\left\{K_{g 1}\right.$, Nonce $\left._{1}\right\} K_{13}$ and $\left\{K_{g 1}\right.$, Nonce $\left._{1}\right\} K_{14}$ are sent to members 2, 3 and 4 respectively. Finally, the latter decrypt the received message and store the group key before they respond with the acknowledgement messages: $\left\{\right.$ Ack, Nonce $\left._{1}+1\right\} K_{12},\left\{\right.$ Ack $_{,}$Nonce $\left._{1}+1\right\} K_{13}$ and $\left\{\right.$ Ack, Nonce $\left._{1}+1\right\} K_{14}$ respectively. 


\subsubsection{New node addition phase}

To add a new node $N n$ to the network, the gateway node $G N i$ that is close to this new node, randomly generates a new positive secret value $S i$ and broadcasts it to its neighboring nodes encrypted with the group key. Each node that receives this message, decrypts it with the group key, extracts and saves the secret value, and encrypts a message in which it inserts the new secret value $S i$ and transmits it to its direct neighbors. This process is repeated until all the nodes obtain this new value. This value and the hash function $\operatorname{Hash}()$ are also stored in the memory of the new node $N n$ before deploying it in the network so that they will be used to calculate the secret keys that the new node will share with its neighbors.

$$
G N i \rightarrow *:\{\text { Si, Nonce } i\} K_{g i}
$$

where $K_{g i}$ is the group key.

After deployment, the new node broadcasts a join message along with its identifier, a random number (nonce) and a digest.

$$
\left.N n \rightarrow *: \text { Join, Idn } \| \text { Nonce }_{n}, \text { Hash(Idn } \| \text { Nonce }_{n}, \text { Si }\right)
$$

The adjacent devices that are within the radio range of that new node, receive it then check its authenticity and integrity. If this verification fails, the message will be ignored, otherwise all the neighboring nodes will update their neighbors vector by adding the new node's identifier $I d n$, and their matrices will also be updated by creating, at the end, a new row and a new column, where the elements are equals to zero except the main diagonal element that equals to $M_{j}[k+1, k+1]=$ Nonce $_{n}$, with $k$ represents the order of the square matrix $M_{j}$ just before adding the new node. After that, each neighboring node $N j$ will respond with an acknowledgement message that contains its diagonal element $M[j, j]$ of the matrix $M$ and the group key $K_{g i}$.

$N j \rightarrow N n: A c k, I d j \|$ Nonce $_{j}\|M[j, j]\|$ Nonce $_{n}+1 \| K_{\text {gi }}$, Hash $_{\text {Idj }} \|$ Nonce $_{j}\|M[j, j]\|$ Nonce $\left._{n}+1 \| K_{\text {gi }}, S i\right)$

Upon the reception of the last message, the new node checks it. If this verification is successful, the new node stores the group key and updates its neighbors vector by adding the identifier of node $N j$. When this neighbor vector is completly updated, the new node updates its square matrix in the following way: $M n[i, j]=M[v n[i], v n[i]]$, with $i=j, M n[i, j]=0$, otherwise.

When the matrix $M n$ is fully updated, the new node can start to generate all the symmetric keys $K n i$ that will be shared with each of its neighbor $N i$ by executing the step $b$ ) of the pairwise key establishment phase. After that, it replies to the node $N j$ with a message containing the succesor of the received nonce Noncej, encrypted with the obtained pairwise key $K n j$.

$$
N n \rightarrow N j:\left\{\text { Nonce }_{j}+1\right\} K n j
$$

At the end, the node $N j$ responds to the gateway node $G N i$ with the message that contains the succesor of the nonce of $N i$ encrypted with the pairwise key $K_{i j}$.

$$
N j \rightarrow N i:\left\{\text { Nonce }_{i}+1\right\} K_{i j}
$$

The process of adding a new node to the network is described in the Figure 4 below. 


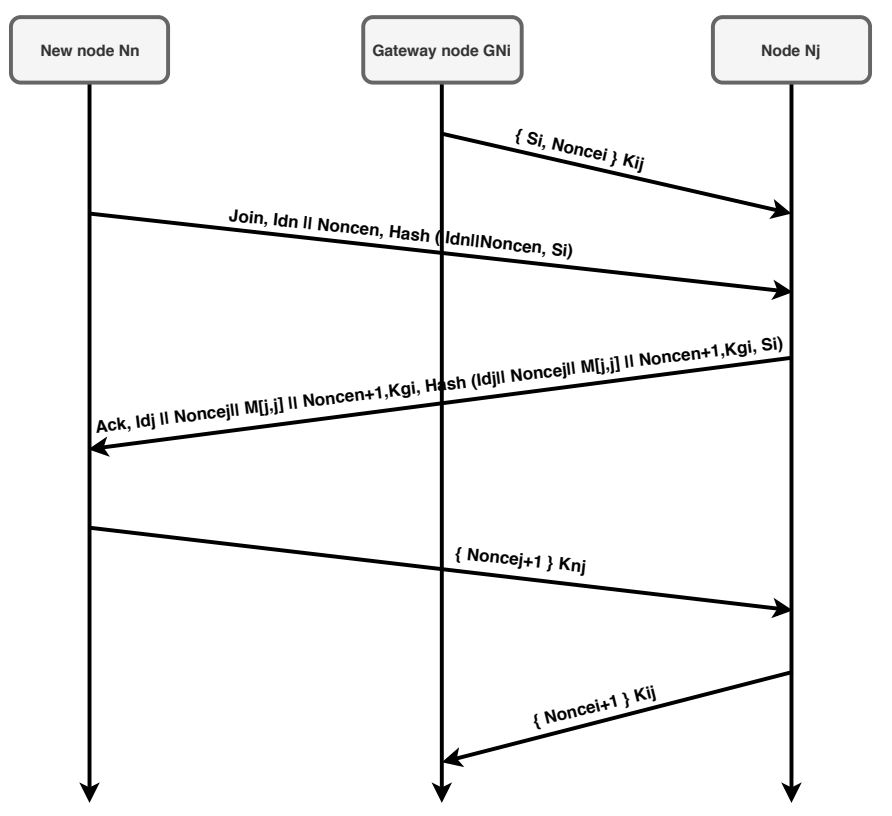

Figure 4: New node addition process

\section{Example}

The Figure 5 shows an example of adding a new node to the network. The network is initially composed of six nodes. The gateway node 1 and the node 2 are neighbors to each others and have the same neighbor (node 3 ), therefore, they share the same neighbors vectors $v i=[1,2,3]$, with $i=1,2$, and the same square Matrix $M_{i}=\left[\begin{array}{lllllll}1 & 2 & 3 ; 4 & 5 & 6 ; 7 & 8 & 9\end{array}\right]$, with $i=1,2$. The node 4 wants to join the network. So, the gateway node 1 close to this new node, generates a random secret value $S 1$ and sends it to its neighboring nodes 2 and 3 encrypted with the group key $K_{g 1}$. The new node 4 is preloaded with this secret value $S 1$ and the hash function $\operatorname{Hash}()$ before its deployment, then it generates a random number(Nonce 4 equals to 55 for example) and sends a same join message to its 1 hop neighbors (node 1 and 2). The nodes 1 and 2 check the authenticity of the join message by computing the hash value of the first part of the message and compares it with the second part. If the verification succeed, then both nodes add the identifier 4 to their neighbors vectors $v i=[1,2,3,4]$, with $i=1,2$. After that the nodes 1 and 2 add a new forth row where $M_{i}(4, j)=0$, with $i=1,2$ and $j=1,2,3$ and $M_{i}(4, j)=$ Nonce $4=55$ else (with $i=1,2$ and $j=4$ ), and a new forth column where $M_{i}(j, 4)=0$, with $i=1,2$ and $j=1,2,3$ and $M_{i}(j, 4)=$ nonce $4=55$ else (with $i=1,2$ and $j=4$ ). The nodes 1 and 2 respond with the acknowledgement messages containing the group key $K_{g 1}$ and the diagonal elements $M(1,1)=1$ and $M(2,2)=5$, respectively. Upon the reception of the $A C K$ messages, the new node 4 ckecks if they are authentic. if yes then it saves the group key $K_{g 1}$ and adds the identifiers 1 and 2 to its neighbors vector $v 4=[1,2,4]$ ordered and updates its square matrix $M 4=\left(\begin{array}{lllllll}1 & 0 & 0 ; 0 & 5 & 0 ; 0 & 0 & 55\end{array}\right)$. The new node 4 has its 3 by 3 matrix fully updated, so it is able to generate the symmetric keys $K 14$ and $K 24$ by executing the step $b$ ) of the pairwise key establishment phase as follows:

The node 1 and 4 compute the secret value:

$S 14=|\operatorname{det}(M 14)|=\left|\operatorname{det}\left(\begin{array}{lll}1 & 0 ; 0 & 55\end{array}\right)\right|=|55|=55$, and then generate the common key: $K 14=\operatorname{Hash}(1 \|$ $4 \| 55,55)=\operatorname{Hash}(1455,55)$.

The node 2 and 4 compute the secret value:

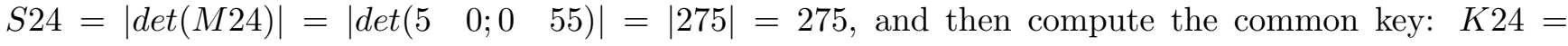
$\operatorname{Hash}(2\|4\| 275,275)=\operatorname{Hash}(24275,275)$

The new node 4 sends to node 1 and 2 the message containing the succesor of the nonce of node 1 and 2 encrypted with the corresponding pairwise key $K 14$ and $K 24$ respectively.

Finally, the node 2 replies to the gateway node 1 with the message containning the successor of the 
received nonce encrypted with the symmetric key $K 12$.

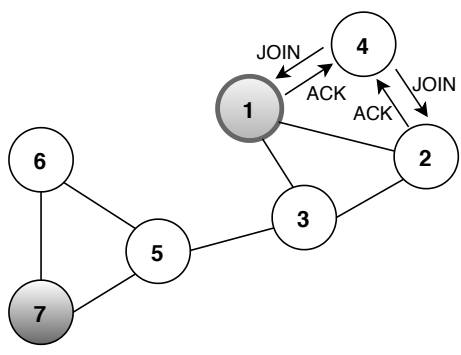

a) Before

$\mathrm{Vi}=[1,2,3] ; \mathrm{Mi}=[123 ; 456 ; 78$ 9], with $\mathrm{i}=1,2$

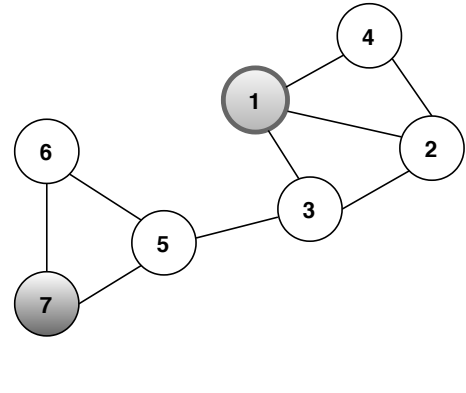

b) After

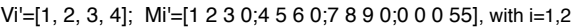

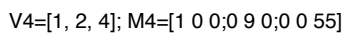

Figure 5: Example of addition of node 4 to the network

\subsubsection{Key revocation phase}

Two cases may arise: either the node leaves after it has been detected as compromised, or it leaves with its proper willingness.

Case 1: A gateway node $G N i$ that detects a bad or suspicious behavior (does not forward correctly messages for example) from its neighboring node $N j$ does the following tasks:

a) deletes from its memory the pairwise key that it shares with it.

b) updates its square matrix by deleting the row and the column that correspond to that node.

c) updates its neighbors vector by removing the identifier of the compromised node.

d) executes the group key establishment phase in order to generate a new group key $K_{g i}^{\prime}$ by using the pairwise keys of the remaining members.

e) informs its nearby nodes $N k$ by sending them a message encrypted with the appropriate pairwise keys. The message contains the identifier of the compromised node, a nonce and a new group key.

$$
G N i \rightarrow N k:\left\{I d j \| \text { Nonce }_{i}, K_{g i}^{\prime}\right\} K_{i k}
$$

When a node receives the last message that comes from its close gateway, it does the following actions:

1. decrypts it with the appropriate pairwise key and replaces the old group key by the new one.

2. compares the identifier received with its identifier and with the identifier of the sender. If it is different from both identifiers then it checks if the compromised node belongs to its neighbors. In other words, it verifies whether the identifier of the compromised node appears in its neighbors vector. If it is not the case, the message will be ignored by the recipient, otherwise the node does the operations $a$ ), $b$ ) and $c$ ) described above.

3. replies to the sender by an acknowledgment message Ack encrypted with the appropriate symmetric key that contains the successor of the received nonce.

$$
N k \rightarrow G N i:\left\{\text { Ack }_{\text {Nonce }}+1\right\} K_{i k}
$$


Case 2: A node $N i$ that leaves the network with its own will, broadcasts a Leave message encrypted with the group key in order to inform its 1-hop neighbors.

$$
N i \rightarrow *:\left\{\text { Leave, Idi } \| \text { Nonce }_{i}\right\} K_{g i}
$$

A node $N j$ who receives that message, decrypts it with the group key, responds with an acknowledgment message Ack and carries out the operations $a$ ), b) and $c$ ) described above. The gateway node executes the task $d$ ) on top of the three operations $a$ ), b) and $c$ ).

$$
N j \rightarrow N i:\left\{\text { Ack, Idj } \| \text { Nonce }_{i}+1\right\} K_{j i}
$$

All the neighboring nodes delete the pairwise keys that they shared with the outgoing or compromised node. As a result, the latter can no longer communicate with its neighbors afterwards. In other words, the neighbors of the leaving or compromised node will avoid to transmit messages to him since they dont hold appropriate symmetric keys.

\section{Example}

We consider the network depicted in the Figure 6 below. The node 2 leaves the network with its own will, so it informs its neighboring nodes (node 1 and 4) by broadcasting a Leave message encrypted with the group key $K_{g 1}$.

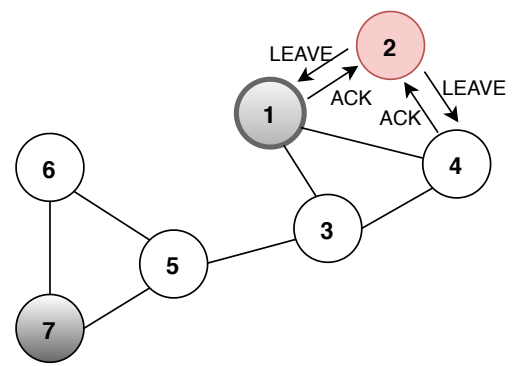

a) Before

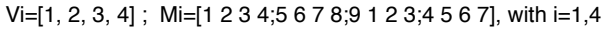

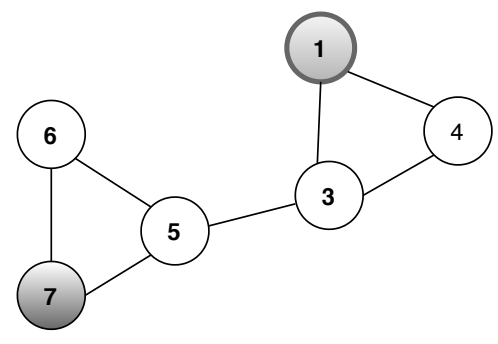

b) After

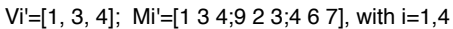

Figure 6: Example of deletion of node 2 from the network

Before the departure of the node 2, we assume that the neighbors vectors and the square matrices of the node 1 and 4 are equals to:

$$
v 1=v 4=[1,2, \quad 3,4] ; M 1=M 4=\left[\begin{array}{llll}
1 & 2 & 3 & 4 \\
5 & 6 & 7 & 8 \\
9 & 1 & 2 & 3 \\
4 & 5 & 6 & 7
\end{array}\right]
$$

When receiving the Leave message, the nodes 1 and 4 do the following operations:

- decrypt the message with the group key $K_{g 1}$.

- reply to the leaving node by an Ack messages: $\left\{\right.$ Ack, $1 \|$ Nonce $\left._{2}+1\right\} K 12$ and $\left\{\right.$ Ack, $4 \|$ Nonce $\left._{2}+1\right\} K_{2} 24$ respectively.

- delete from their memories the pairwise keys $K 12$ and $K 24$ respectively.

- update their square matrices $M 1$ and $M 4$ by deleting the second row and the second column that correspond to the node 2 .

- update their neighbors vectors $v 1$ and $v 4$ by deleting the identifier 2 of the node 2 .

After the deletion of the node 2, the neighbors vectors and the square matrices of the nodes 1 and 4 become: 
$v^{\prime} 1=v^{\prime} 4=\left[\begin{array}{lll}1, & 3,4\end{array}\right] ; M^{\prime} 1=M^{\prime} 4=\left[\begin{array}{lll}1 & 3 & 4 \\ 9 & 2 & 3 \\ 4 & 6 & 7\end{array}\right]$

Furthermore, the gateway node 1 reruns the group key establishment phase so as to renew the old group key and sends it, in a secure manner, to nodes 3 an 4 within a messages encrypted with the pairwise keys $K_{13}$ and $K_{14}$ respectively.

\subsubsection{Key refresh phase}

This phase aims to improve security because the new keys will be completly different from the old ones, which add difficulties to cryptanalytic attack. In fact, if the same keys are kept for a long time, the attacker may be able to obtain these keys by traffic analysis. Therefore, all the keys should be updated. The refresh process is initiated periodically by the gateway nodes after the expiration time is reached. This process can be devided into two subphases that are detailled as follows:

a) The goal of this first subphase is that the neighboring gateway nodes agree about a small secret value. During this subphase, each gateway node $G N i$ generates a random positive number $S i$ as secret and it sends the message below, encrypted with the appropriate pairwise key $K_{i j}$, to its 1-hop neighboring gateway node $G N j$.

$$
G N i \rightarrow G N j:\{\text { Refresh, Idi } \| S i\} K_{i j}
$$

Upon the reception of the above message, the recipient uses the appropriate pairwise key $K_{i j}$ to decrypt it. After that, it extracts and saves the secret value Si in its list ist $_{j}:=L_{i s t}+$ Si $_{\text {. }}$

At the end of this subphase, each gateway node $G N i$ determines the smalest secret value Min $_{i}$ among all the values belonging to its list.

$$
\operatorname{Min}_{i}=\min \left(S_{k}\right), \text { with } \quad S_{k} \in \text { List }_{i} .
$$

b) In this second subphase, each gateway node $G N i$ perfoms the following operations:

1. generates a nonce Nonce $_{i}$ and broadcasts the following refresh message that contains the smallest secret value $\mathrm{Min}_{i}$, encrypted with the current group key $K_{g i}$.

$$
G N i \rightarrow *:\left\{\text { Refresh }_{\text {Idi }} \| \text { Nonce }_{i} \| \operatorname{Min}_{i}\right\} K_{g i}
$$

2. updates its square matrix by multiplying the main diagonal elements times the smalest positive value $\operatorname{Min}_{i}$. So $M_{j}[k, k]=\operatorname{Min}_{i} * M_{j}[k, k] \quad$ with $\quad k=1, . ., n$, and $n$ represents the order of the square matrix $M_{j}$.

3. executes the step $b$ ) of the pairwise key establishment phase in order to generate new symmetric keys, which can be used for further communication.

4. carries out the group key establishment phase to generate a new group key $K_{g i}^{\prime}$.

5. deletes the old or prior keys from its memory and replaces them with the new ones.

Each constrained node who receives the above refresh message for the first time, it uses the current group key $K_{i j}$ to decrypt it, then it extracts the secret value and executes the steps 2) 3) and 5) above. After that, it replies to the sender by the following acknowledgment message, which contains the successor of the received nonce, encrypted with the new pairwise key. Note that constrained nodes that have more than one gateway node nearby, accept the first refresh message received and disregard the others.

$$
N j \rightarrow G N i:\left\{A c k, I d j \| \text { Nonce }_{i}+1\right\} K^{\prime} i j
$$




\section{Security analysis}

In this section, we first give an informal analysis of our scheme by showing its resistance against several types of attacks. After that, we present a formal analysis by using AVISPA (Automated Validation of Internet Security Protocols and Applications) tool [17].

\subsection{Informal analysis}

The proposed approach is resilient to the following types of attacks.

- Eavesdropping attack

IoT devices generally use wireless links to communicate with each other. An adversary can easily listen to messages when they are clearly transmitted over wireless links.

In our scheme, during the key generation phase between two neighbors, an adversary can have access to the first part of the Hello message because it is not encrypted. However, he is not able to compute the symmetric pairwise key since he knows neither the initial square matrix M nor the one way hash function Hash() that are used. After the key estalishment phase, all communications betwen nodes are secured with the established pairwise and group keys. As a result, an attacker could not read the content of exchanged messages. Thus, our system is resilient to this kind of attack.

- Compromising (Node capture) attack

An adversary who captures and compromises an IoT device can have access to all the information stored in its memory (secret keys, square matrix and the hash function). A node stores one group key and at most a number of pairwise keys equals to the number of its neighbors. Therefore, the attacker can only read, alter and/or delete messages transmitted over the communication channels shared between the compromised node and its direct neighbors. The other channels will not be affected and will remain safe. We assume that this type of attack does not occur before the deletion of initial square matrix M, otherwise an adversary will be able to compute all the secrets keys. In other words, if the initial matrix $\mathrm{M}$ is compromised before the expiration of the timer then all the secret keys will be revealed. However, if an adversary captures and compromises a node after the expiration of the timer, then only the node's symmetric keys will be discovered because the initial matrix $\mathrm{M}$ has been deleted from its memory. On the other hand, an adversary that captures a gateway node dont have access to the information stored in its memory since it is protected by the use of TPM $[15,16]$. Thus, the communication links betwen the gateway nodes and the server could not be compromised.

- Sybil attack

In a Sybil attack, a malicious node provides several identities to other nodes in the network. To protect against such attack, the identity of each node needs to be checked. This can be performed directly by a neighboring node or indirectly by another trusted entity.

In our system, this verification is performed directly by a neighboring node since a node shares a unique symmetric key with each of its neighbors. So every node checks the identity of its neighbor by using the appropriate symmetric key when they want to establish a link betwen them. Thus, a malicious node cannot claim to be another node without prior knowledge of all the secret keys stored in the memory of the latter. On the other hand, in neighbor discovery and join phases for example, a node inserts its identity then generates a MAC (Message Authentication Code), which is added to the message before sending it. The recipient then uses this MAC to verify the identity and authenticate the sender. Therefore, the proposed system offers a certain robustness against this attack.

- Forward and backward secrecy

Forward secrecy ensures that a node is unable to decipher new messages by using an old key. As 
for backward secrecy, it is the opposite. That means, a node is unable to decrypt previous messages encrypted with old keys by using the new key. Both forward and backward secrecy are used to defeat node capture attacks.

In the proposed scheme, when a node is compromised or leaves the network, all its neighbors remove from their memories the symmetric key that they shared with it and update their neighbor vectors and square matrices. Therefore, the leaving node cannot use the old symmetric keys to establish a new communication with its neighbors because these latter have already removed this old shared key from their memories. Furthermore, these neighbors cannot establish any more new communications with this leaving node since they cannot generate a new secret key for this latter because of the deletion of the row and the column corresponding to this node from their square matrices. A node that has left the network is unable to decrypt the new messages by using the old keys, because all its neighborings nodes have removed these keys from their memories. Similarly, when a node joins the network again, it can not decipher the previous messages, because the new keys differ from the old ones. Thus, our scheme ensures forward and backward secrecy.

- Replay attack

In a replay attack, the attacker first does passive eavesdropping by capturing the data exchanged over the network, and later uses this obsolete data to violate integrity or authentication.

Freshness is introduced into our scheme by using a random and unpredictible numbers such as secret values and nonces in order to defeat this kind of attack. In addition, an attacker is not able to reuse the messages of an old session in another new session since keys are periodically refreshed. Of course, in the key refresh phase for instance, if the attacker does passive eavesdropping and captures the refresh message sent by a gateway node $G N i$ to its constrained nodes, the attacker will not be able to decrypt that message since he does not know the group key $K_{g i}$. When the attacker replays such rekeying message captured in the previous session, the recipient node $N j$ remarks that the new group key $K_{g i}^{\prime}$ does not match with the old one, with which the message is encrypted. In fact, the node $N j$ has already received a fresh message comming from the close gateway node $G N i$ or from another one, and thus it has already updated its pairwise and group keys and deleted the old ones from its memory. As a result, the recipient node detects such replay attack.

- Man in the middle attack

In a man in the middle attack, the attacker is placed between two communicating entities in order to intercept and/or modify the exchanged messages. The sender and the recipient are unaware of the existance of the middleman.

In our scheme, all the messages are exchanged directly between any pair of IoT neighbors without any intermediate entity. Both IoT nodes detect the presence of the adversary since they receive the messages sent by this unauthorised user. Furthermore, only Hello and Join messages are not encrypted, but they contain $M A C$ part so that any modification of the messages during their transmission will be detected by the recipient. All of the other exchanged messages in our scheme are encrypted with the appropriate keys, so the attacker is not able neither to read nor to alter their content. In others words, the attacker can not launch any attack on confidentiality and integrity. Thus our scheme is robust against man in the middle attack.

Furthermore, our scheme ensures the following properties that must be taken into consideration to assess key management protocols:

- Extensibility since it allows new devices to be added to the network after the deployment phase by going through the node addition phase. Thus, our scheme is flexible and allows dynamism.

- Scalability because each node stores one group key and at most only the pairwise keys of its neighbors, thus sensors are assigned with a small number of keys to save their memories. As a 
result, the number of keys stored in a constrained node's memory does not increase linearly or exponnentially with the add of new devices.

- Resilience since the corruption of a given node has limited consequences on the whole of the system. Indeed, an attacker who compomises a node will only have access to the information (keys) stored in its memory and as the node stores few keys (one group key and at most only these of its direct neighbors), by consequence such an attack will have little influence on the whole of the system. In other words, if a given node is compromised, less number of keys will be revealed to the adversary.

- Authentication through the use of initial shared secret such as hash function, initial matrix and nonces in the key management.

- Distribution since the initial context used in the key establishment process is distributed in an offline mode before the deployment.

On the other hand, the secret keys are established in a distributed manner, thus avoiding a central entity that could be a point of failure or weakness.

\subsection{Formal analysis}

In this subsection, we give a formal analysis of our scheme by using AVISPA tool [17]. AVISPA (Automated Validation of Internet Security Protocols and Applications) is a modeling tool for building and analyzing formal models of the security protocols. It includes four back-ends: OFMC (On-the-fly Model-Checker)[18], CL-AtSe (Constraint-Logic-based Attack Searcher)[19], SATMC (SAT-based ModelChecker) [20] and TA4SP (Tree Automata based on Automatic Approximations for the Analysis of Security Protocols).

Our protocols are first written in Alice-Bob notation then specified in High Level Protocol Specification Language HLPSL [21]. After that their security properties like secrecy, integrity and authentication are analyzed by the four back-ends of the AVISPA tool.

As an example, the Alice-Bob notation for the JOIN protocol is given below.

1. $S->A:\{S s, N s\} K s a$

2. $B->A: B, N b, \operatorname{Hash}(B, N b, S s)$

3. $A->B: A, N a, S a, S u c c(N b), K g, \operatorname{Hash}(A, N a, S a, S u c c(N b), K g, S s)$

4. $B->A:\{\operatorname{Succ}(N a)\} K a b$

5. $A->S:\{\operatorname{Succ}(N s)\} K s a$

where $S$ is a gateway node, $A$ a node and $B$ a new node.

The obtained results show that all protocols are SAFE under the OFMC and CL-AtSe, INCONCLUSIVE under SATMC and SAFE or INCONCLUSIVE under TA4SP back-ends. That means that the security goals stated in the environment roles (secrecy, integrity and authentication) are satisfied and could not be violated. In other words, the back-ends found no attacks as shown in Table 2. 


\begin{tabular}{lllll}
\hline Phase/Back-end & OFMC & CL-AtSe & SATMC & TA4SP \\
KEP & $\begin{array}{l}\text { Version of 2006/02/13 } \\
\text { SUMMARY } \\
\text { SAFE }\end{array}$ & $\begin{array}{l}\text { SUMMARY } \\
\text { SAFE }\end{array}$ & $\begin{array}{l}\text { SUMMARY } \\
\text { INCONCLUSIVE }\end{array}$ & $\begin{array}{l}\text { SUMMARY INCONCLIVE } \\
\text { JOIN }\end{array}$ \\
& SUMMARY & SUMMARY & SUMMARY & SUMMARY \\
& SAFE & SAFE & INCONCLUSIVE & INCONCLUSIVE \\
LEAVE & SUMMARY & SUMMARY & SUMMARY & SUMMARY \\
SAse1 & SAFE & SAFE & INCONCLUSIVE & INCONCLUSIVE \\
LEAVE & SUMMARY & SUMMARY & SUMMARY & SUMMARY \\
RAse2 & SAFE & SAFE & INCONCLUSIVE & SAFE \\
REFESH & SUMMARY & SUMMARY & SUMMARY & SUMMARY \\
& SAFE & SAFE & INCONCLUSIVE & SAFE \\
\hline
\end{tabular}

Table 2: Results of protocols specifications verified in AVISPA

Therefore, we can affirm that an attacker is not able to launch an attack against the authentication, the integrity and the secrecy on our protocols. Of course, the mutual authentication betwen a node and its neighbors is satisfied by exchanging their nonces. The secrecy of the sensitive data, like shared key is also guaranteed. The integrity is guaranteed by using hash function. In fact, for instance when an intruder intercepts the message $M, \operatorname{Hash}(M, k)$ and substitutes the message $M$ by $\dot{M}$, he will not be able to compute $\mathrm{H}\left(\hat{M}^{\prime}, \mathrm{k}\right)$ since he does not know the secret key $K$. Whenever he sends $\dot{M}, \mathrm{H}(\mathrm{M}, \mathrm{k})$, the recipient immediately notices that the message has been modified since the hash value of the received message $\mathrm{H}\left(\mathrm{M}^{\prime}, \mathrm{k}\right)$ is different from the hash value sent $H(M, k)$.

\section{Performance analysis}

In this section, we first make a comparative study of the solutions presented in related work section. After that, we evaluate the performance of our scheme by simulations. We especially estimate the energy consumption of constrained node as Mica2dot sensor node since its energy capacity is very limited. We mainly focus on communication and computation overheads during pairwise key establishment and key refresh phases.

\subsection{Comparative study}

A comparative study of our scheme with the key establishment protocols seen in related work section according to the memory (storage), computation and communication requirements during key establishment phase, is shown in Table 3 below.

In our scheme, each node needs to store a group key and at most only the pairwise keys of its direct neighbors. The gateway nodes also store their public/private keys and the public key of the server node. In addition, a node also stores a square matrix of order $d$ ( $d$ is the number of neighbors) and a neighbors vector of $d$ elements. Thus, one node stores $d(d+1)$ elements. If the size of one element is four bytes, then the total space required to store the key material is $4 * d(d+1)$. In the network of 1000 nodes for instance, each node has about 14 neighbors according to [22]. Hence, one node needs to store 840 bytes that is negligible compared with the capacity of the very constrained node like Mica2dot, which is about $512 \mathrm{kB}$.

In the other hand, our scheme needs less computation than the others. In fact, to compute one pairwise key, a node carries out only two multiplications and one substraction (addition), unlike most of the other schemes which require at least $\lambda+1$ multiplications and $\lambda$ additions and, it depends on the security parameter $\lambda$.

Furthermore, to establish one pairwise key betwen a constrained node and a gateway node, they need to exchange only two Hello messages, whereas in Messai et al scheme, to compute one secret key between a sensor and its cluster head, the sensor node has to send one and receive $d$ Hello messages from its neighbors. 


\begin{tabular}{|c|c|c|c|}
\hline Schemes & Memory & Computation & Communication \\
\hline Blom [5] & $2(\lambda+1)$ & $\begin{array}{l}(\lambda+1) \text { multiplications } \\
\lambda \text { additions }\end{array}$ & $\begin{array}{l}\operatorname{Col}_{j}(G) \\
(\lambda+1)\end{array}$ \\
\hline Du et al $[6]$ & $\begin{array}{l}1+t \cdot(\lambda+1) \\
t \text { key space }\end{array}$ & $\begin{array}{l}2 \lambda+1 \text { multiplications } \\
\lambda \text { additions }\end{array}$ & $\begin{array}{l}\text { the seed of } G \\
\text { and indices of } t\end{array}$ \\
\hline Yu et al [7] & $\begin{array}{l}(\lambda+1)(2+w) \\
w \text { rows of } B\end{array}$ & $\begin{array}{l}\lambda+1 \text { multiplications } \\
\lambda \text { additions }\end{array}$ & $\operatorname{Col}_{j}(G)$ \\
\hline Rahman et al. [8] & $(\lambda+2)$ & $\begin{array}{l}2 \lambda+1 \text { multiplications } \\
\lambda \text { additions }\end{array}$ & - \\
\hline Y. Zhang et al [9] & key seed $s i$ & $\begin{array}{l}2 \lambda+1 \text { multiplications } \\
\lambda \text { additions }\end{array}$ & $\begin{array}{l}\text { request and } \\
\operatorname{row}_{i}\left(A_{c}\right)\end{array}$ \\
\hline Messai et al [12] & $\begin{array}{l}(n+4) \text { keys } \\
n: \text { number of cluster members }\end{array}$ & $\begin{array}{l}M A C+ \\
\text { Encryption }\end{array}$ & $\begin{array}{l}(d+1) \text { Hello } \\
\text { messages }\end{array}$ \\
\hline Mesmoudi et al [13] & $(n+2)$ keys & $\begin{array}{l}M A C+ \\
\text { Encryption }\end{array}$ & $\begin{array}{l}\text { Hello }+ \text { Hello_Rep } \\
\text { messages }\end{array}$ \\
\hline Our scheme & $\begin{array}{l}d(d+1) \\
d \text { neighbors }\end{array}$ & $\begin{array}{l}2 \text { multiplications } \\
1 \text { substraction }+M A C\end{array}$ & $\begin{array}{l}2 \text { Hello } \\
\text { messages }\end{array}$ \\
\hline
\end{tabular}

Table 3: Comparative study of the schemes

\subsection{Experimental study (Simulation results)}

We perform simulations by using the MATLAB environment to assess the performance of our scheme with respect to the two related protocols, EAHKM+ [12] and SKWN [13] . In the simulation, we consider a network in which nodes are randomly deployed within a square area of $100 \mathrm{~m} \times 100 \mathrm{~m}$. The number of nodes varies from 10 to 100 . We assume that the rate of gateway nodes and cluster heads is $10 \%$ of the network size. The communication range of nodes is $10 \mathrm{~m}$. We use the energy model presented in [23]. In this model, the cost of receiving one byte is about $(28.6 \mu J)$ and sending a byte is about $(59.2 \mu J)$ for the Mica2dot node.

In this subsection, we present the average obtained results for 1000 runs of the simulation in each case. We first evaluate the resilience of our scheme against node capture attack. After that, we estimate the comunication and computation energy consumed by constrained nodes during both pairwise key establishment and key refresh phases.

\subsubsection{Resilience against node capture}

The resilience is computed as the fraction of links compromised $F x$ when $x$ nodes are captured. In our scheme, when an adversary captures a constrained node, he could read all the keys stored in its memory. Since each node stores a group key and, at most, the symmetric keys of its neighbors, the number of links that will be compromised is equal to twice the number of its neighbors (the degree $d$ of the compromised node) because when an attacker gets the symmetric key $K_{i j}$ for example, he is able to decrypt all messages exchanged between nodes $i$ and $j$ in both directions, i.e from $i$ to $j$ and vice versa. However, if a gateway node is captured, the number of links that will be compromised is zero because the adversary gets no key since the memory of this type of node is protected by a TPM $[15,16]$. Figure 7 shows the obtained results in terms of resilience to node capture attack of our scheme. We assume that the adversary compromises one to twenty constrained nodes randomly, and the network considered is composed of 1000 nodes (constrained and gateways nodes). We note that the proportion of compromised links increases with increasing the number of compromised nodes, which remains negligible in our protocol. 


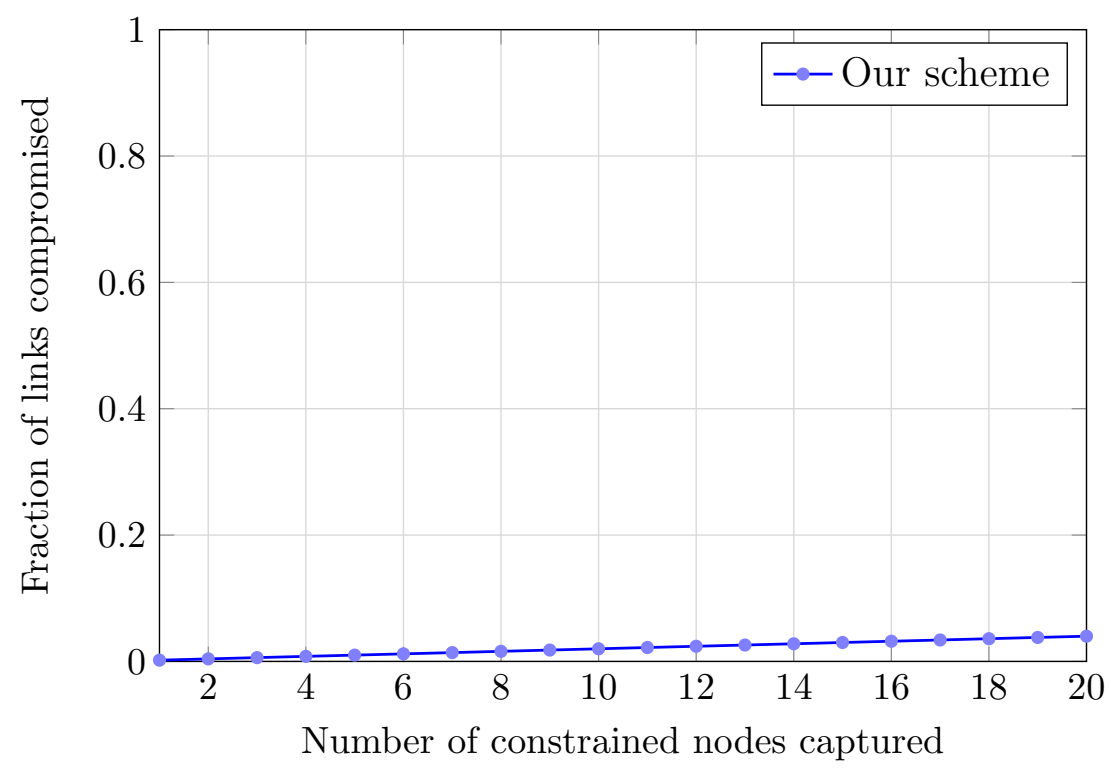

Figure 7: Resilience to node capture attack

\subsubsection{Communication overhead}

Since the communication cost is closely related on the number and the size of each message exchanged betwen two communicating neighboring nodes, we first evaluate the length of each message sent or received by a constrained node during the differents phases of our scheme, then we give the estimation of energy consumption by sending or receiving such messages according to the energy model given in [23], after that we discuss the simulation results. We assume that the size of the parameters within messages are 1 byte for message type, 4 bytes for identity of node, 4 bytes for nonce, $\mathrm{E}$ bytes for encryption and $\mathrm{H}$ bytes for hash value. The energy consumption on communications of our scheme is given in Table 4 .

\begin{tabular}{|c|c|c|c|c|}
\hline Phase & $\begin{array}{c}\text { Message } \\
\text { sent }\end{array}$ & $\begin{array}{l}\text { Message } \\
\text { received }\end{array}$ & $\begin{array}{l}\begin{array}{l}\text { Length } \\
\text { (byte) }\end{array} \\
\end{array}$ & $\begin{array}{l}\text { Energy consumption } \\
(\mu J)\end{array}$ \\
\hline Init & - & - & - & - \\
\hline KEP & (1) & (2) & $\begin{array}{l}1+4+4+\mathrm{H}=9+\mathrm{H} \\
\mathrm{d}^{*}(1+4+4+\mathrm{H})=\mathrm{d}^{*}(9+\mathrm{H})\end{array}$ & $\begin{array}{l}(9+\mathrm{H}) * 59.2 \\
\mathrm{~d}^{*}(9+\mathrm{H}) * 28.6\end{array}$ \\
\hline JOIN & $\begin{array}{l}(12) \\
(14)\end{array}$ & $\begin{array}{l}(10) \\
(11) \\
(13)\end{array}$ & $\begin{array}{l}\mathrm{E} \\
1+4+4+\mathrm{H} \\
1+4+4+4+4+16+\mathrm{H} \\
\mathrm{E} \\
\mathrm{E}\end{array}$ & $\begin{array}{l}\mathrm{E}^{*} 28.6 \\
(9+\mathrm{H})^{*} 28.6 \\
(33+\mathrm{H}) * 59.2 \\
\mathrm{E}^{*} 28.6 \\
\mathrm{E}^{*} 59.2\end{array}$ \\
\hline LEAVE & (16) or (18) & $(15)$ or $(17)$ & $\begin{array}{l}\mathrm{E} \\
\mathrm{E}\end{array}$ & $\begin{array}{l}\mathrm{E}^{*} 28.6 \\
\mathrm{E}^{*} 59.2\end{array}$ \\
\hline REFRESH & (21) & (20) & $\begin{array}{l}\mathrm{E} \\
\mathrm{E}\end{array}$ & $\begin{array}{l}\mathrm{E}^{*} 28.6 \\
\mathrm{E}^{*} 59.2\end{array}$ \\
\hline
\end{tabular}

Table 4: Length of messages sent or received by a constrained node

Figure 8 illustrates the average communication energy (in joules) consumed by all constrained nodes during pairwise key establishment phase in function of hash algorithm. We note that the communication overhead increases for the compared schemes when the size of hash value increases. As the energy consumption is closely related to the amount of data exchanged, the quantity generated during this phase depends on the hash algorithm used and therefore on the size of hash value. We notice that this quantity is more important in SKWN and EAHKM+ than in our scheme. As a result, the performance of our scheme are higher. 


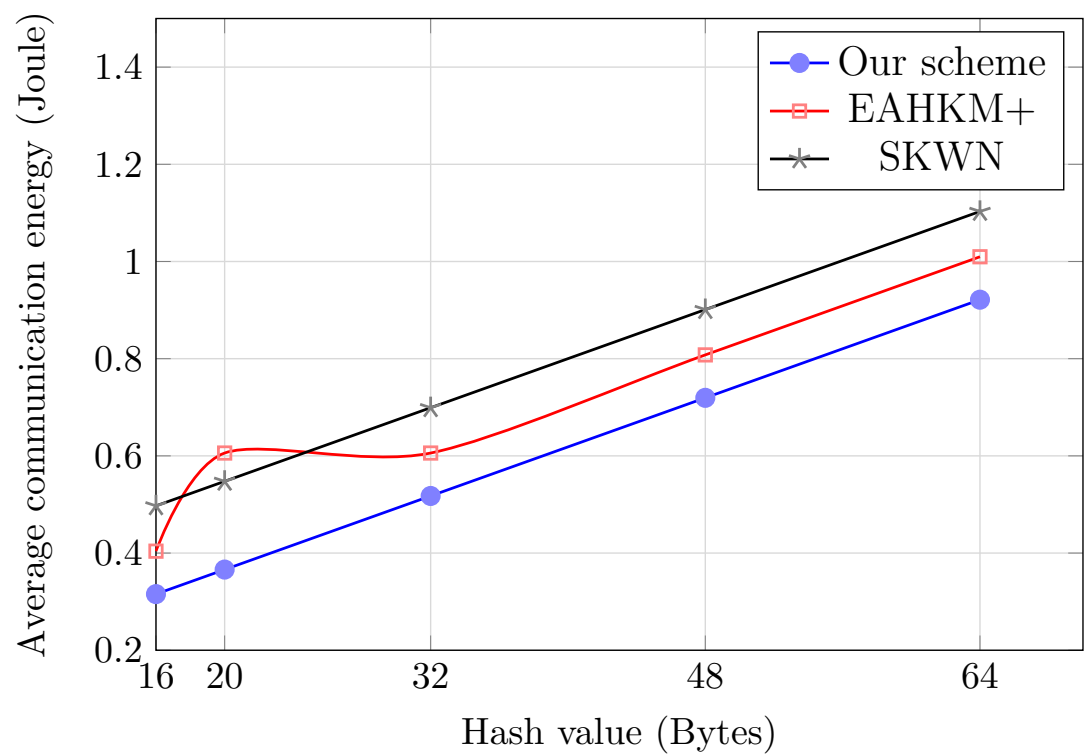

Figure 8: Communication energy consumed by constrained nodes during pairwise key establishment

Figure 9 presents the average energy (in joule) dissipated in communication by all constrained nodes during pairwise key establishment phase according to the network size. As shown in this Figure, the communication cost of our scheme is better than the other protocols. Although the number of messages exchanged during this phase in our scheme and EAHKM+ is the same, but their size in EAHKM+ is greater than ours. As we know, the energy consumption depends directly on the amount of data sent and/or received.

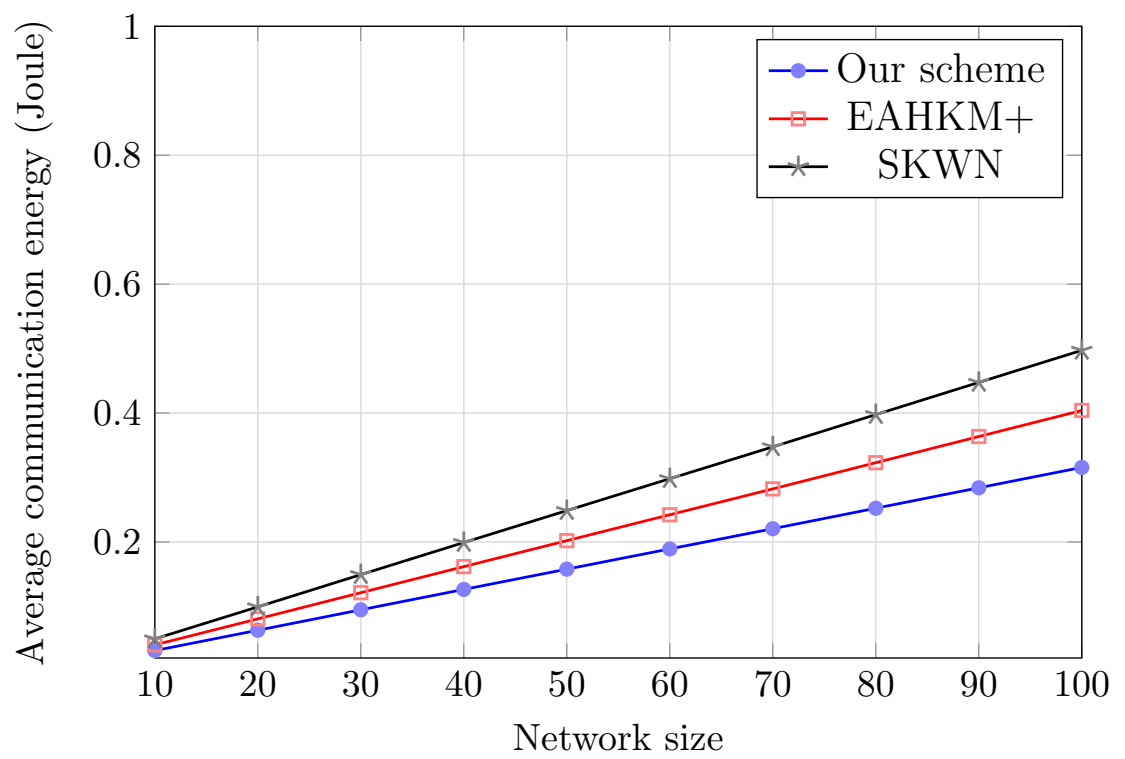

Figure 9: Communication energy consumed by constrained nodes during key establishment

Figure 10 plots the average communication energy (in joule) consumed by all constrained nodes during key refresh phase in function of the network size. We assume that the encryption algorithm used here is AES-128 and the size of hash value is 16 bytes. This Figure indicates clearly that our scheme saves more energy than the other protocols. This is because in our system, during the rekeying process, each 
constrained node only needs to receive few encrypted message while in EAHKM + for instance, each sensor must receive $(d+1)$, which significantly increases the communication overhead. Moreover, we notice that the consumed energy increases more slowly in our scheme than in EAHKM+ and SKWN. This can be explained by the fact that the quantity of messages received by sensors, in both schemes EAHKM+ and SKWN, depends mainly on the number of neighbors or cluster members, which also depends on the network size.

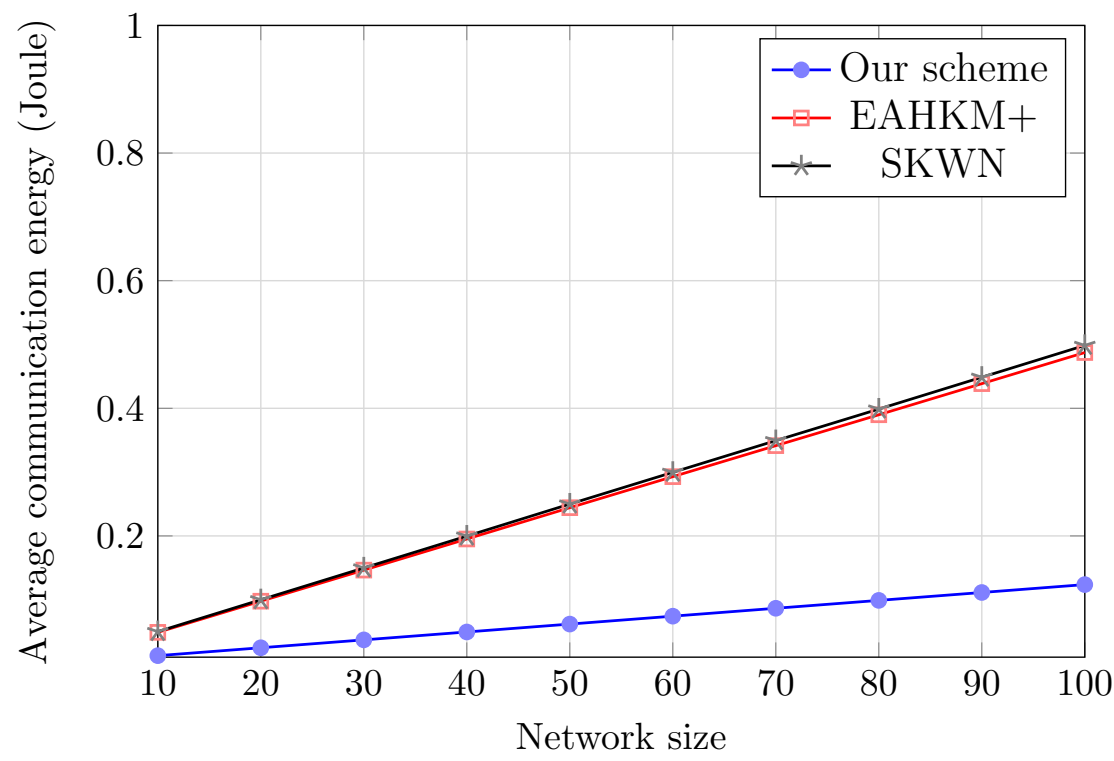

Figure 10: Communication energy consumed by constrained nodes during key refresh

\subsubsection{Computation overhead}

The computation overhead mainly depends on encryption, decryption and authentication cost. To evaluate this cost, we consider the total number of encryption, decryption and hash operations performed by a constrained node in each phase of our scheme. After that, we estimate the energy consumption, then we discuss the simulation results. According to authors in [14], the energy cost of one AES-128 encryption (or decryption) and hash operation in TelosB platform is $9 \mu J$ and $40 \mathrm{~mJ}$ respectively. The obtained results are summerized in Table 5.

\begin{tabular}{llll}
\hline Phase & $\begin{array}{l}\text { Number of AES-128 } \\
\text { encryption (decryption) }\end{array}$ & $\begin{array}{l}\text { Number of Hash } \\
\text { operations }\end{array}$ & $\begin{array}{l}\text { Energy } \\
\text { consumption }(\mu \mathrm{J})\end{array}$ \\
\hline Init & 0 & 0 & 0 \\
KEP & 0 & $1+2 * \mathrm{~d}$ & $(1+2 * d) * 40 * 10^{3}$ \\
JOIN & $1+(1+1)$ & 2 & $03 * 9+2 * 40 * 10^{3}$ \\
LEAVE & $01+(01)$ & 0 & $02 * 9=18$ \\
REFRESH & $01+(01)$ & 0 & $02 * 9=18$ \\
\hline
\end{tabular}

Table 5: Number of encryption, decryption and hash operations performed by a constrained node

Figure 11 plots the average energy consumption (in joules) during the computation of the pairwise keys shared betwen constrained nodes with their gateway nodes in our scheme and sensors with their cluster heads in EAHKM+ and SKWN. Our scheme outperforms the others and our results are slightly better than those of EAHKM+ and SKWN. This is because, in our scheme, constrained nodes only perform hash operations to establish pairwise keys, but in the other protocols, sensors do encryption and decryption in addition to hash operations, which increases slightly the computation overhead. 


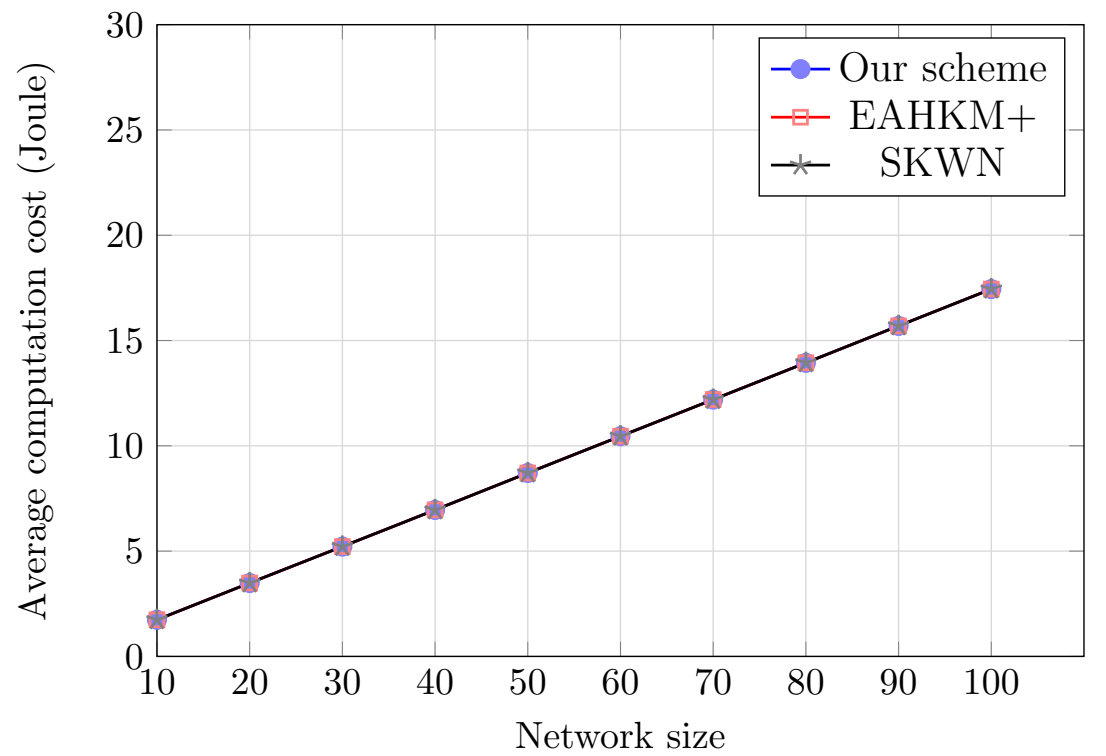

Figure 11: Computation cost in the constrained node side during key establishment

Figure 12 compares the computation cost in terms of the average energy consumed by all constrained nodes during key refresh phase in function of the network size. In our scheme, we considered the worst case where each constrained node computes all the new pairwise keys of its neighbors, while in the schemes EAHKM+ and SKWN, each sensor computes only one key with its cluster head. In spite of this, the obtained results show that our scheme consumes less energy than the other protocols. Indeed, in EAHKM+ and SKWN, sensors carry out more encryption, decryption and hash operations. Furthermore, in our scheme to update keys, constrained nodes only reexecute the step $b$ ) of pairwise key establishment phase, but in EAHKM+ for example, sensors rerun the whole cluster formation and key establishment algorithm.

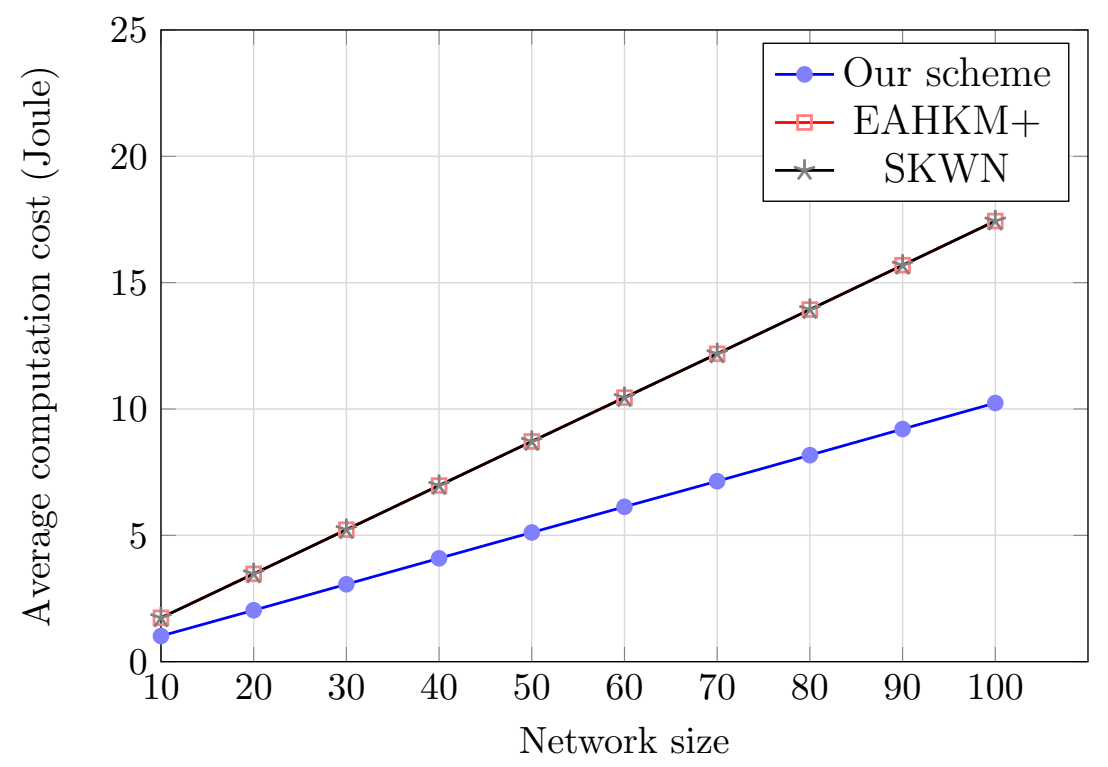

Figure 12: Computation cost in the constrained node side during key refresh 


\section{Conclusion}

In this paper, we have proposed a lightweight matrix based key management scheme for securing communications betwen IoT devices. The proposed scheme uses matrix to allow any node to be able to compute a distinct pairwise key with its neighbors. Security analysis shows that the proposed scheme ensures the security goals like secrecy, integrity and authentication and can protect the sensitive data from various types of attacks. In addition, the proposed system allows extensibility, scalability, resilience, authentication and distribution. Furthermore, the simulation results show that our scheme resists well to node capture attack and consumes less energy when compared to two recent protocols proposed in the literature.

As future work, we propose to explore the use of blockchain technology in order to improve performance and achieve better efficiency of our scheme. In fact, the blockchain can allow the network members to authenticate each others without going through an intermediate entity. For example, during new node addition phase, members will be able to authenticate new nodes without the need of gateway nodes. In other words, the addition process will be decentralized.

\section{References}

[1] S. Li, L. Da Xu, S. Zhao, The internet of things: a survey, Information Systems Frontiers 17 (2015) $243-259$.

[2] S. L. Keoh, S. S. Kumar, H. Tschofenig, Securing the internet of things: A standardization perspective, IEEE Internet of things Journal 1 (2014) 265-275.

[3] Z. Bi, L. Da Xu, C. Wang, Internet of things for enterprise systems of modern manufacturing, IEEE Transactions on industrial informatics 10 (2014) 1537-1546.

[4] R. Roman, C. Alcaraz, J. Lopez, N. Sklavos, Key management systems for sensor networks in the context of the internet of things, Computers \& Electrical Engineering 37 (2011) 147-159.

[5] R. Blom, An optimal class of symmetric key generation systems, in: Workshop on the Theory and Application of of Cryptographic Techniques, Springer, pp. 335-338.

[6] W. Du, J. Deng, Y. S. Han, P. K. Varshney, J. Katz, A. Khalili, A pairwise key predistribution scheme for wireless sensor networks, ACM Transactions on Information and System Security (TISSEC) 8 (2005) 228-258.

[7] Z. Yu, Y. Guan, A key management scheme using deployment knowledge for wireless sensor networks, IEEE Transactions on Parallel and Distributed Systems 19 (2008) 1411-1425.

[8] M. Rahman, S. Sampalli, An efficient pairwise and group key management protocol for wireless sensor network, Wireless Personal Communications 84 (2015) 2035-2053.

[9] Y. Zhang, Y. Xiang, X. Huang, X. Chen, A. Alelaiwi, A matrix-based cross-layer key establishment protocol for smart homes, Information Sciences 429 (2018) 390-405.

[10] L. Xu, Y. Zhang, Matrix-based pairwise key establishment for wireless mesh networks, Future Generation Computer Systems 30 (2014) 140-145.

[11] Q. Wang, H. Su, K. Ren, K. Kim, Fast and scalable secret key generation exploiting channel phase randomness in wireless networks, in: 2011 Proceedings IEEE INFOCOM, IEEE, pp. 1422-1430.

[12] M.-L. Messai, H. Seba, Eahkm+: energy-aware secure clustering scheme in wireless sensor networks, International Journal of High Performance Computing and Networking 11 (2018) 145-155. 
[13] S. Mesmoudi, B. Benadda, A. Mesmoudi, Skwn: Smart and dynamic key management scheme for wireless sensor networks, International Journal of Communication Systems 32 (2019) e3930.

[14] G. De Meulenaer, F. Gosset, F.-X. Standaert, O. Pereira, On the energy cost of communication and cryptography in wireless sensor networks, in: Networking and Communications, 2008. WIMOB'08. IEEE International Conference on Wireless and Mobile Computing,, IEEE, pp. 580-585.

[15] W. Arthur, D. Challener, A practical guide to TPM 2.0: using the Trusted Platform Module in the new age of security, Apress, 2015.

[16] S. L. Kinney, Trusted platform module basics: using TPM in embedded systems, Elsevier, 2006.

[17] A. Armando, D. Basin, Y. Boichut, Y. Chevalier, L. Compagna, J. Cuéllar, P. H. Drielsma, P.C. Héam, O. Kouchnarenko, J. Mantovani, et al., The avispa tool for the automated validation of internet security protocols and applications, in: International conference on computer aided verification, Springer, pp. 281-285.

[18] D. Basin, S. Mödersheim, L. Vigano, An on-the-fly model-checker for security protocol analysis, in: European Symposium on Research in Computer Security, Springer, pp. 253-270.

[19] M. Turuani, The cl-atse protocol analyser, in: International Conference on Rewriting Techniques and Applications, Springer, pp. 277-286.

[20] A. Armando, L. Compagna, Satmc: a sat-based model checker for security protocols, in: European workshop on logics in artificial intelligence, Springer, pp. 730-733.

[21] Y. Chevalier, L. Compagna, J. Cuellar, P. H. Drielsma, J. Mantovani, S. Mödersheim, L. Vigneron, A high level protocol specification language for industrial security-sensitive protocols, in: Workshop on Specification and Automated Processing of Security Requirements-SAPS'2004, Austrian Computer Society, pp. 13-p.

[22] H. Chan, A. Perrig, D. Song, Random key predistribution schemes for sensor networks, in: Security and Privacy, 2003. Proceedings. 2003 Symposium on, IEEE, pp. 197-213.

[23] A. S. Wander, N. Gura, H. Eberle, V. Gupta, S. C. Shantz, Energy analysis of public-key cryptography for wireless sensor networks, in: Pervasive Computing and Communications, 2005. PerCom 2005. Third IEEE International Conference on, IEEE, pp. 324-328. 